This is a preprint of Fluid phase equilibria 217.1 (2004): 53-57. The original article canbe found under http://www.sciencedirect.com/science/article/pii/S0378381203004989

\title{
Prediction of vapor liquid equilibria using COSMOtherm
}

\author{
By Andreas Klamt* and Frank Eckert \\ COSMOlogic GmbH\&CoKG, Burscheider Str. 515, 51381 Leverkusen, Germany \\ * correspondence author, Tel.: +49-2171-731681, e-mail: klamt@cosmologic.de
}

keywords: molecular simulation; method of calculation; activity coefficients; phase diagrams; COSMO-RS

\begin{abstract}
The COSMO-RS method in its COSMOtherm implementation has been used to predict the vapor liquid equilibria of problem sets $1 \mathrm{a}$ and $1 \mathrm{~b}$ of the first industrial fluid property prediction challenge, i.e. for the mixtures of dimethylether (1) and propene (2) and of nitroethane (1) and propylene glycol monomethyl ether (1-methoxy2-propanol) (2). Good quality predictions for both systems have been achieved within less than one day of work, using experimental values for the pure compound vapor pressures and predicted activity coefficients for the mixture thermodynamics. In addition to the results reported for the challenge predictions based on predicted pure compound vapor pressures are reported and discussed in this paper.
\end{abstract}

\section{Introduction}

The a priori prediction of the thermodynamic behavior of mixtures of new compounds or of new mixtures of well-known compounds is a longstanding industrially important problem. Questions of this kind often appear in chemical engineering when new processes or alternatives for existing processes are considered, and in product design and development, when the quality or performance of a product depends on the solubility or partition behavior of compounds between different liquid phases. Traditionally two very different approaches have been followed for the prediction of fluid phase equilibrium properties. From the chemical engineering side group contribution methods like UNIFAC [1] have been developed, which allow for an interpolation of the fluid phase equilibrium properties, if sufficient information is available for other compounds which have essentially the same functional groups. But because of their oversimplifying basic assumption of additivity, these methods are not capable of reflecting any more specific intra- and intermolecular interactions, like electronic push-pull effects, intramolecular hydrogen bonding, conformational ambiguity, etc. Therefore there are many situations where group contributions cannot satisfactorily predict the behavior of mixtures which involve more complicated compounds with several functional groups. A second and much more fundamental approach has been chosen by the molecular modeling community [2-5]. This approach tries to build rigorous, physically realistic models of the liquid systems by considering large ensembles of virtual molecules in periodic boxes and studying the averages of free and internal energy and of other properties either by molecular dynamics or by Monte Carlo simulations. Due to the availability of powerful supercomputers such simulations can nowadays be done within reasonable time. The basic problem of this approach is the need to use severe approximations for the description of the intra- and intermolecular interactions, the so-called force fields. Typically such force-fields have a lot parameters which are specially optimized for certain areas of chemistry or for the prediction of special properties. While powerful force-fields have been developed for non-polar compounds, which meanwhile allow for an almost perfect reproduction of the phase diagrams of alkanes mixtures [3-5], the extension of these force-fields towards the inclusion polar interactions and of hydrogen bonding causes large problems. Therefore the predictive power of the MD/MC approach is still quite limited for general mixtures of organic compounds.

During the last 5 years the COSMO-RS method [6-9] has been established as an efficient alternative to the two previous approaches. It starts directly from quantum chemical calculations for the individual molecules and expresses the intermolecular interactions based on this quantum chemical information. The combination of this relatively fundamental description of intermolecular interactions with a fast and accurate statistical thermodynamics of interacting surfaces led to an efficient and broadly applicable new approach to the prediction 
of the thermodynamic properties of liquid mixtures. Despite of some limitations, it is applicable in many situations where he other methods fail. The first industrial fluid phase simulation challenge has been the first occasion for an objective comparison of the predictive capabilities of this novel approach with the traditional methods.

\section{The COSMO-RS method}

The name COSMO-RS is composed of "COnductor-like Screening MOdel" (COSMO) [10], which is an efficient variant of dielectric continuum solvation methods in quantum chemical programs, and its extension to "Real Solvents" (RS)[6], which is a statistical thermodynamics approach based on the results of quantum chemical COSMO calculations. Since the full description of the COSMO-RS theory is beyond the scope this article and has been given in several recent articles [7-9], we restrict ourselves to a short introduction of the basic features required for the understanding this article.

COSMO-RS is a two step procedure. In a first step, quantum chemical calculations have to be performed for all compounds of interest. In these calculations the continuum solvation model COSMO is applied in order to simulate a virtual conductor environment for the molecule. In this environment, the solute molecule induces a polarization charge density $\sigma$ on the interface of the molecule to the conductor, i.e. on the molecular surface, and these charges act back to the solute, generating a more polarized electron density than in vacuum. During the quantum chemical self-consistency algorithm the solute molecule is thus converged to its energetically optimal state in a conductor with respect to electron density and geometry. The standard quantum chemical method for COSMO-RS is density functional theory (DFT) and the DFT functional B88-PW86 [11,12] with a triple zeta valence polarized basis set (TZVP) is used throughout this study. All DFT/COSMO calculations have been performed using the quantum chemical program TURBOMOLE [13], which is very efficient for this kind of calculation. The molecules considered in this contest were relatively small. Hence none of the calculations for the 4 molecules took longer than $12 \mathrm{~min}$. on a single $800 \mathrm{MHz}$ CPU. All further details and references regarding DFT/COSMO calculations with TURBOMOLE are given in ref. [14].

The polarization charge density $\sigma$ is a good local descriptor of the molecular surface polarity. In the second step of COSMO-RS, the statistical thermodynamics of the molecular interactions, this polarization charge density is used for the quantification of the interaction energy of pair-wise interacting surface segments with regard to the most important molecular interaction modes, i.e. electrostatics and hydrogen bonding. The less specific van der Waals (vdW) interactions or dispersive interactions are taken into account in a more approximate way by element specific dispersion coefficients. Because these cancel out in liquid-liquid equilibria, the dispersive interactions are of no importance for the purpose of this paper. Thus the quantum chemical information about the polarization charge densities $\sigma$ plays the key role for the evaluation of the molecular interactions in the liquid phase. As a prerequisite for an efficient statistical thermodynamics treatment, the 3D distribution of the polarization charges $\sigma$ on the surface of each molecule $\mathrm{X}$ is converted into a surface composition function $(\sigma-$ profiles), i.e. a histogram $\mathrm{p}^{\mathrm{X}}(\sigma)$, which gives the relative amount of surface with polarity $\sigma$ on the surface of the molecule. Such $\sigma$-profiles provide detailed information about the molecular polarity distribution [7,8], but they do no longer provide geometric $3 \mathrm{D}$ information.

The statistical thermodynamics itself is done using a coupled set of non-linear equations for the activity coefficients of the surface segments. This new method, which was recently published separately under the name COSMOSPACE (COSMO Segment Activity Coefficient Equations) [15], was specially developed for the COSMO-RS method [6]. It provides an exact solution for independently pair-wise interacting surface segments and thereby has a more realistic behavior, especially at infinite dilution, than the traditional chemical engineering methods, e.g. UNIQUAC, which make additional approximations.

All COSMO-RS calculations of this paper are performed using the COSMOtherm program [16], which provides an efficient and flexible implementation of the COSMO-RS method. The total CPU time spent for the over 1000 COSMOtherm calculations required for this paper took only about $2 \mathrm{~min}$ on a $900 \mathrm{MHz}$ laptop computer. The latest parameterization The parameterization BP_TZVP_C11_0101 is used. In such parameterization the very 
few parameters of the COSMO-RS method, i.e. two parameters for each chemical element and about 10 general parameters, are optimized based on a set of about 1000 thermodynamic data, mainly partition coefficients and vapor pressures [for details see ref. 9], in combination with DFT/COSMO calculations of a certain quantum chemical method. Such parameterization may be considered as the equivalent of a specific force-field in MD/MC simulations, but it has much less adjusted constants than typical force fields, since only global and element specific constants are required in COSMO-RS. Detailed values of the parameterization used in this are given in ref. [9]

\section{Results for Problem Set 1, Part A:}

The two compounds considered in this part are dimethylether (1) and propene (2). Both compounds have a single minimum energy conformation. The corresponding DFT/COSMO calculations led to the $\sigma$-profiles as shown in figure 1. Graphical visualizations of the screening charge densities on the molecular surfaces are provided under ref. [17].

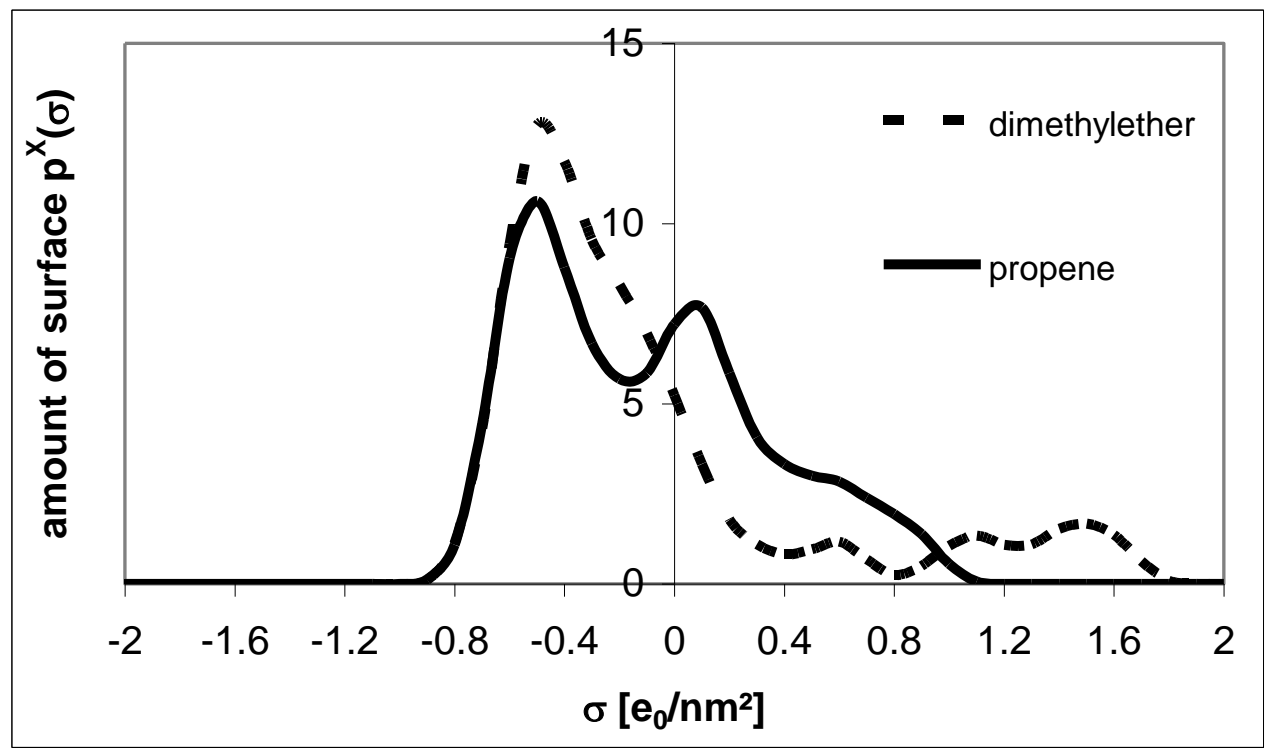

Figure 1: $\sigma$-profiles for compounds in problem set 1 part A.

The vapor mole fractions $y_{i}$ have been calculated from the ratio of partial and total vapor pressure:

$$
y_{i}=p_{i}^{0} x_{i} \gamma_{i} / p_{t o t}
$$

The total pressures $p_{\text {tot }}$ have been obtained from

$$
p_{t o t}=p_{1}^{0} x_{1} \gamma_{1}+p_{2}^{0} x_{2} \gamma_{2}
$$

Where, $p_{i}{ }^{0}$ are the pure compound vapor pressures, $x_{i}$ are the mole fractions of the compound in the liquid phase and $\gamma_{i}$ are the activity coefficients as computed by COSMOtherm, for compounds $i(i=1,2)$. For both compounds of problem set 1 part A, the Wagner equation (3) has been used to estimate the pure compound vapor

\begin{tabular}{|c|c|c|c|c|c|c|c|}
\hline & $A$ & $B$ & $C$ & $D$ & $E$ & $F$ & $T_{M I N}$ \\
\hline dimethyl ether & 5370 & 400.0 & -7.1260 & 1.8171 & -3.10 & -0.9164 & 194 \\
\hline
\end{tabular}
pressures $p_{i}{ }^{0}$. The coefficients for equation (3) for compounds dimethyl ether and propene are given in table 1 . 
propene

Table 1: Wagner equation coefficients in $[\mathrm{kPa}] /[\mathrm{K}]$ according to ref. [18]. The last 2 columns give the temperature range of validity in $[\mathrm{K}]$.

The resulting vapor pressures at $-20{ }^{\circ} \mathrm{C}$ and $20{ }^{\circ} \mathrm{C}$ are listed in Table 2 . For comparison, the results of the pure compound vapor pressure prediction by COSMOtherm are listed as well. The parameterization C1.1_0101 used throughout the contest gives systematically high predictions by about a factor 1.5 for dimethyl ether and by a factor about a factor 1.8 for propene. Although the agreement is not excellent, it is within the expected range of accuracy for a priori predictions of vapor pressures of COSMOtherm. Much better agreement can be achieved with the latest parameterization of COSMOtherm as can be seen in the last two column of table 2 .

Wagner eq. $\quad$ calc. with C1.1_0101 calc. with C1.2_0702

$\begin{array}{lcccccc}\mathrm{T}\left[{ }^{\circ} \mathrm{C}\right] & -20 & 20 & -20 & 20 & -20 & 20 \\ \text { dimethyl ether } & 127 & 519 & 186 & 786 & 120 & 589 \\ \text { propene } & 305 & 1015 & 526 & 1905 & 320 & 1381\end{array}$

Table 2: Interpolated experimental vapor pressures and calculated values [in $\mathrm{kPa}$ ]

Isothermal binary calculations for the mixture dimethyl ether (1) and propene (2) at $\mathrm{T}=-20^{\circ} \mathrm{C}$ and $\mathrm{T}=20^{\circ} \mathrm{C}$ have been done at 30 different binary mixture concentrations. Although the two compounds are considerably different regarding polarity, the results of the COSMOtherm predictions indicate an almost ideal mixture behavior. The infinite dilution activity coefficients have been calculated as $\gamma_{1}^{\infty}=1.09$ and $\gamma_{2}^{\infty}=1.00$ at $-20^{\circ} \mathrm{C}$, and $\gamma_{1}{ }^{\infty}=1.10$ and $\gamma_{2}{ }^{\infty}=1.03$ at $20^{\circ} \mathrm{C}$. Therefore for this system a simple assumption of ideal behavior would have led to almost the same results as the COSMOtherm results reported below. The calculated results at both temperatures are given in figure 2, together with the corresponding experimental data.

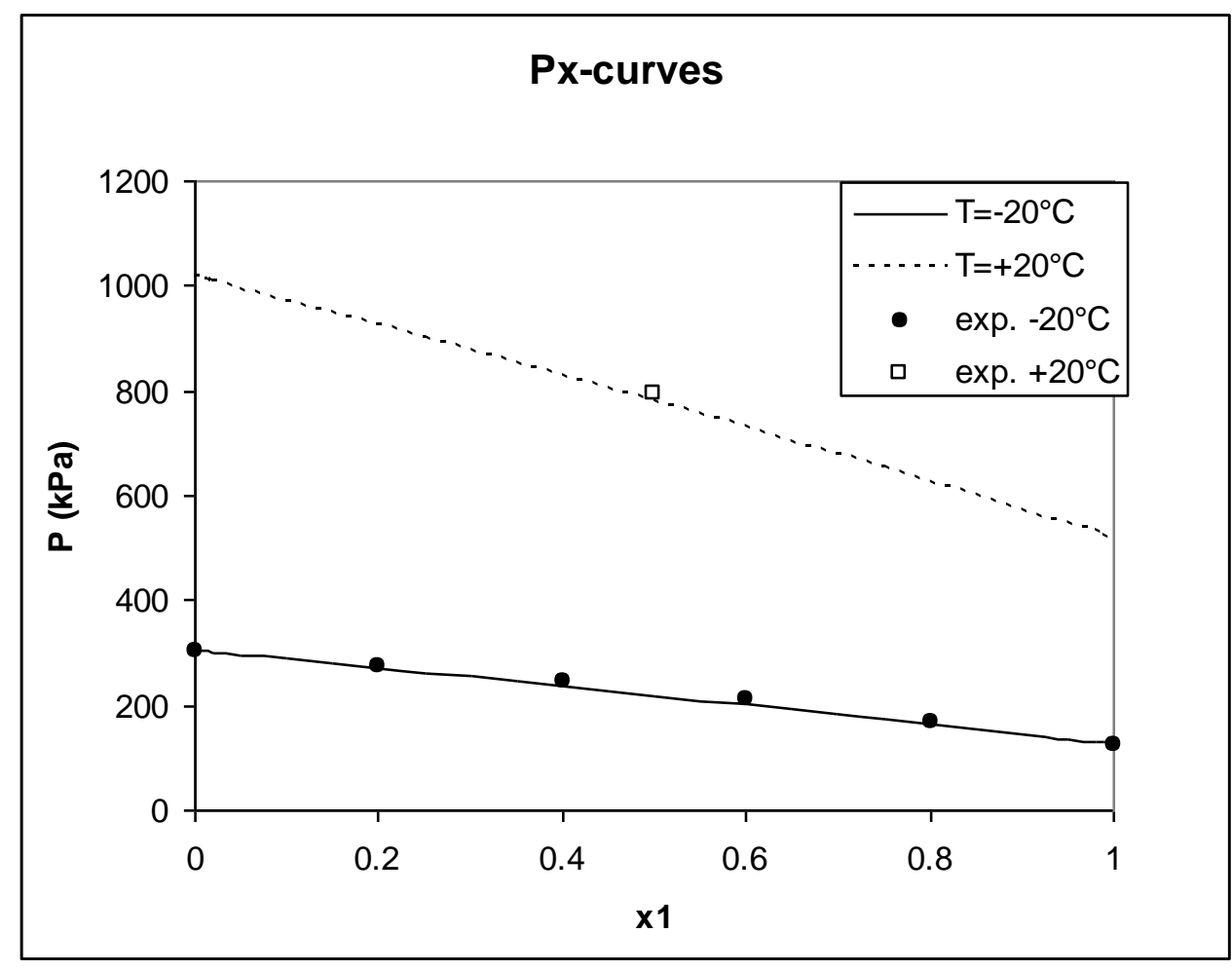

Figure 2: p-x diagram for system dimethyl ether (1) - propene (2) at $\mathrm{T}=-20^{\circ} \mathrm{C}$ and $\mathrm{T}=20^{\circ} \mathrm{C}$. 


\section{Results for Problem Set 1, Part B:}

The two compounds considered in this part are nitroethane (1) and propylene glycol monomethyl ether (1methoxy-2-propanol) (2). Compound (1), nitroethane, is conformationally rather simple. A single minimum energy conformation has been taken into account in the calculation. 1-methoxy-2-propanol (2) has a minimum energy conformation (1-methoxy-2 propanol_conf0) in which the hydroxyl group forms an intramolecular hydrogen bond with the methoxy oxygen. Although being about $6 \mathrm{~kJ} / \mathrm{mol}$ higher in the DFT/COSMO calculation, a second conformation has been taken into account: (1-methoxy-2 propanol_conf1) in which the hydroxyl group is rotated approximately by $120^{\circ}$ and thus is not bound internally. This conformation is more polar, thus it can form more hydrogen bonds externally during the COSMO-RS thermodynamics. Since a third conformation with a hydroxyl rotation of $-120^{\circ}$ is very similar to the second, we did not explicitly calculate it but considered the second conformation to have a degeneracy factor of 2.

The COSMOtherm software allows the representation of a compound by an ensemble of several conformers, each of which being described by an individual COSMO file. If conformations are present, the occupation of the conformations is iterated in an outer self-consistency loop for each temperature and composition, until the occupation is consistent with the chemical potentials including the quantum chemical energy difference. This results in a thermodynamically fully consistent treatment of multiple conformations. The $\sigma$-profiles of nitroethane and for the two conformers of 1-methoxy-2-propanol are shown in figure 3. Colored visualizations of the screening charge densities on the molecular surfaces are provided under ref. [17]

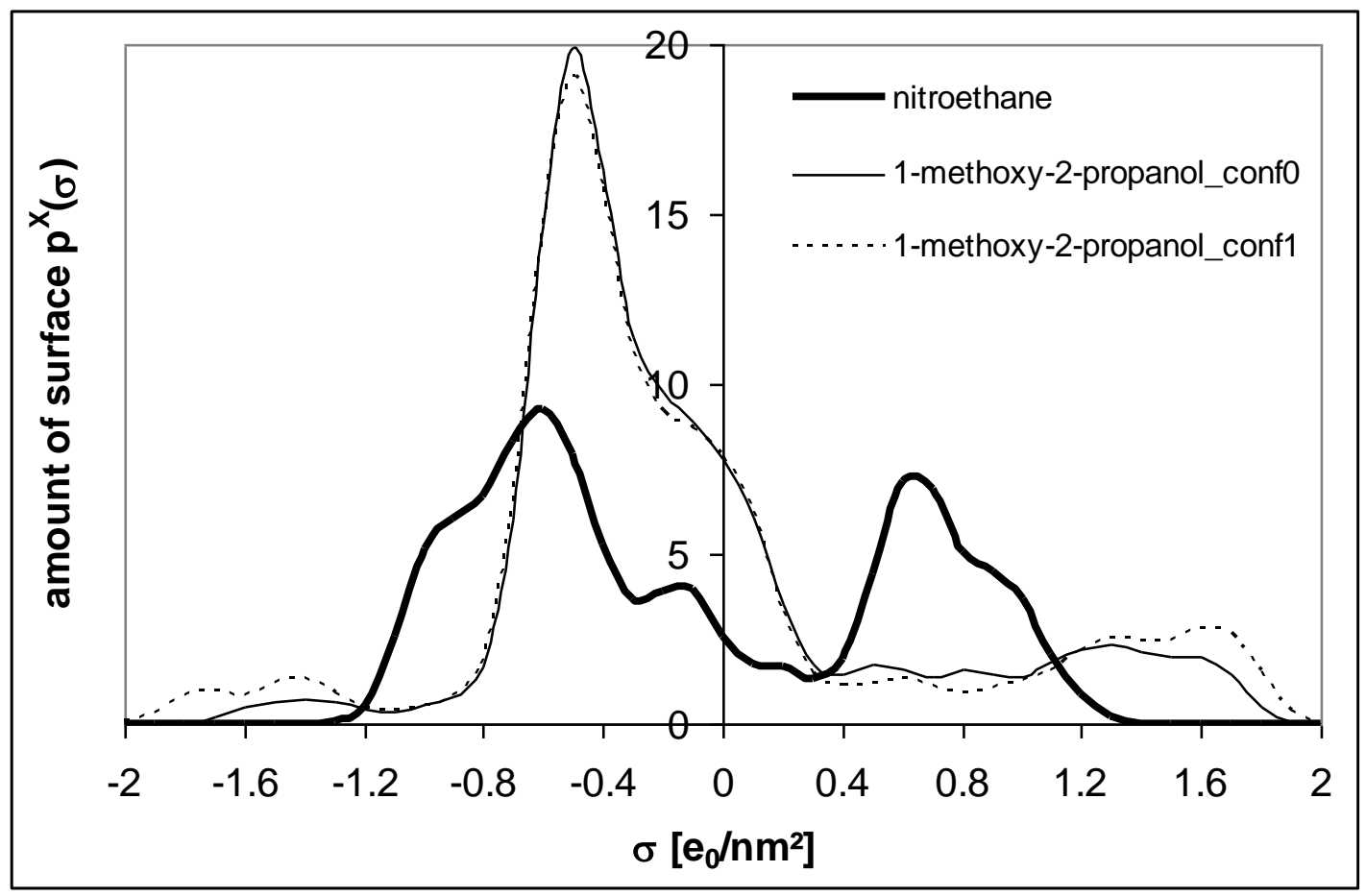

Figure 3: $\sigma$-profiles for compounds in problem set 1 part B.

The vapor mole fractions $y_{i}$ have been calculated in the same way as in Part A, eqs. 1 and 2. The pure compound vapor pressure for nitroethane $p_{1}{ }^{0}$ was computed from experimental data taken from the CRC Handbook of Chemistry and Physics [16]. Here pressures of 1, 10, and $100 \mathrm{kPa}$ are reported for the temperatures 8.3, 50.1 and $113.5^{\circ} \mathrm{C}$, respectively. These three pairs of temperature and pressure data were fitted to the Antoine equation and thus used to obtain pure compound vapor pressures at $40^{\circ} \mathrm{C}$ and $80^{\circ} \mathrm{C}$ that are needed for the COSMOtherm 
VLE calculations. The resulting values are $6.2 \mathrm{kPa}$ and $33.8 \mathrm{kPa}$, respectively. The a priori prediction of vapor pressures using parameterization C1.1_0101 would have lead to values of $15(10.4) \mathrm{kPa}$ at $40^{\circ} \mathrm{C}$ and 68 (47.5) $\mathrm{kPa}$ at $80^{\circ} \mathrm{C}$, respectively. Values of the latest parameterization $\left(\mathrm{C} 1.2 \_0702\right)$ are given for comparison in parentheses.

For compound (2), 1-methoxy-2-propanol we were not able to find reliable pure compound vapor pressure data, apart from a room-temperature value of $1.6 \mathrm{kPa}$ at $25^{\circ} \mathrm{C}$, and a boiling point of $120^{\circ} \mathrm{C}$. Thus, we used the $a$ priori prediction of pure compound vapor pressures of COSMOtherm for this compound. We found an excellent agreement with the experimental data at $25^{\circ} \mathrm{C}\left(\mathrm{C} 1.1 \_0101: 1.6 \mathrm{kPa}\right)$ and $120^{\circ} \mathrm{C}\left(\mathrm{C} 1.1 \_0101: 86 \mathrm{kPa}\right)$ and therefore decided to directly use the COSMOtherm predictions of $3.7(3.5) \mathrm{kPa}$ and $22.8(23.0) \mathrm{kPa}$ for the pure compound vapor pressures at $40^{\circ} \mathrm{C}$ and $80^{\circ} \mathrm{C}$.

Isothermal binary calculations for the mixture nitroethane (1) and 1-methoxy-2-propanol (2) at $\mathrm{T}=40^{\circ} \mathrm{C}$ and $\mathrm{T}=$ $80^{\circ} \mathrm{C}$ have been done at 30 different binary mixture concentrations, using the standard binary grid of the COSMOtherm software. The requested bubble point pressures are given in Table 2. A graphical comparison of the calculated and experimental values is given in Figure 4. Apart from a small and almost constant underestimation of the vapor pressures by about $5 \%$, there is an excellent agreement between the calculated and experimental results. The constant underestimation might result from inaccuracies in the pure compound vapor pressures derived from the Antoine fit and from the COSMOtherm prediction, respectively.

\begin{tabular}{ccccc} 
& \multicolumn{2}{c}{ Predicted C1.1_0101 } & \multicolumn{2}{c}{ experimental } \\
$\mathbf{T}\left[{ }^{\circ} \mathbf{C}\right] \backslash \mathbf{x}_{\mathbf{1}}$ & $\mathbf{0 . 2}$ & $\mathbf{0 . 5}$ & $\mathbf{0 . 2}$ & $\mathbf{0 . 5}$ \\
40 & 4.8 & 5.7 & 4.92 & 5.96 \\
80 & 27.3 & 31.4 & 29.36 & 33.54
\end{tabular}

Table 3: Calculated and experimental bubble point pressures (in $\mathrm{kPa}$ ) at the temperatures and concentrations requested by the contest.

The azeotropic points of the two binary phase diagrams were determined from a graphical plot of the difference of the vapor composition $y_{1}$ and the liquid composition $x_{l}$ as a function of $x_{1}$. In such plot the function $y_{i}-x_{i}$ is zero for an azeotropic point. An azeotrope was found at $x_{l}=0.92$ at $40^{\circ} \mathrm{C}$ and at $x_{1}=0.935$ at $80^{\circ} \mathrm{C}$. The corresponding pressures are $6.2 \mathrm{kPa}$ and $33.9 \mathrm{kPa}$, respectively. Experimentally the azeotropic points were found at $x_{l}=0.825$ at $40^{\circ} \mathrm{C}$ and at $x_{l}=0.765$ at $80^{\circ} \mathrm{C}$. There is an apparent discrepancy between the predicted and experimental positions of the azeotropic points. Presently we do not have a clear explanation for this difference.

The relative importance of the second conformation of 1-methoxy-2-propanol turned out to be quite small. In pure 1-methoxy-2-propanol it is populated by about $12 \%$, while in pure nitroethane the population turns out to be about $4 \%$, with slight changes due to temperature.

\section{Conclusions}

A priori predictions for the vapor liquid equilibria of the two binary mixtures dimethylether and propene, and nitroethane and 1-methoxy-2-propanol have been performed using the COSMO-RS technique in its COSMOtherm implementation. Standard procedures for the these calculations have been used as described in the COSMOtherm manual. No problems occurred throughout the calculations. Since the molecules considered in this paper are relatively small, the total time for the prediction of both VLEs was only half a day. When experimental pure compound vapor pressures are used in the 3 cases where these were available, good qualitative and quantitative agreements are found with the experimental mixture data, which were not available at the time of the calculations.

The quantitative success combined with the relatively low computational and time requirements clearly demonstrate that COSMOtherm is an efficient and reliable tool for the prediction of VLE data for typical industrial relevant mixtures. 
Total Pressure (bar)

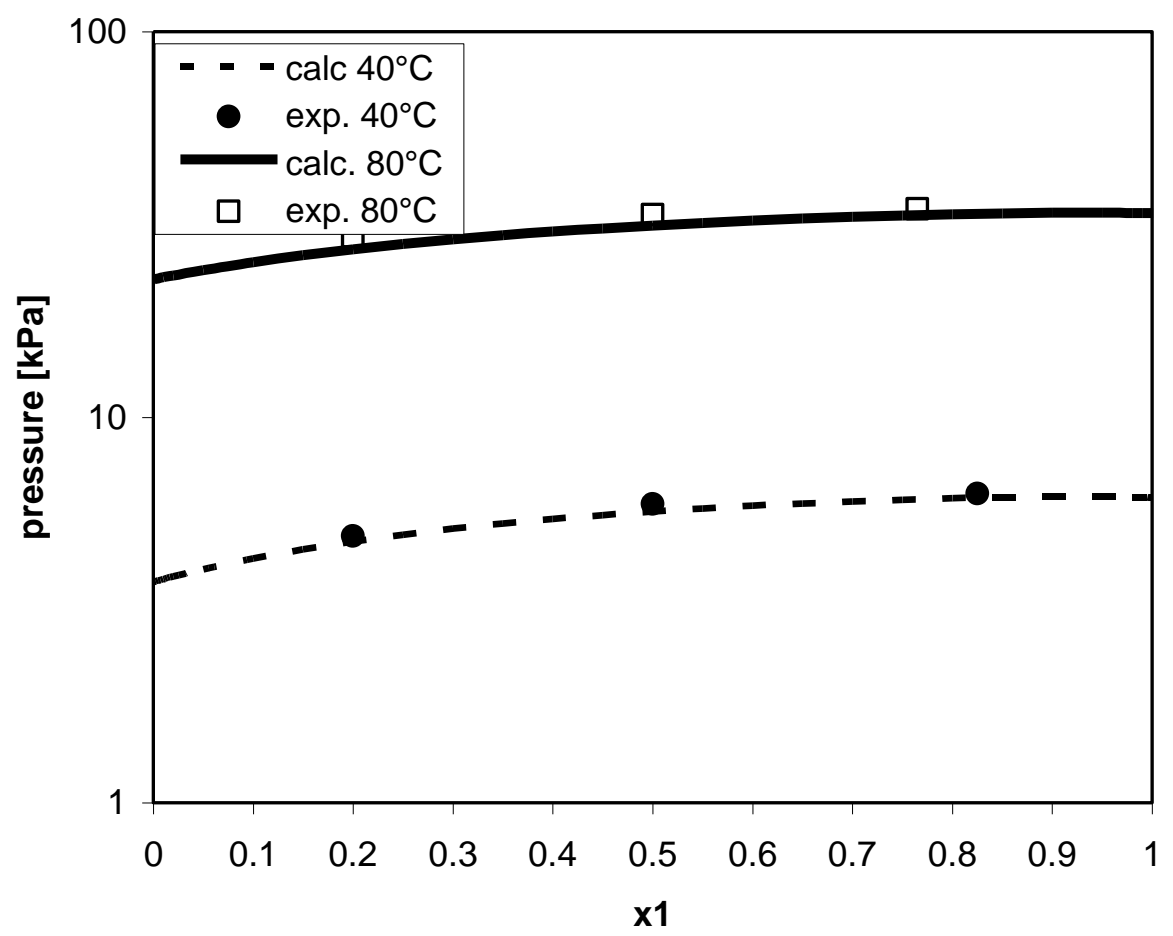

Figure 4: Comparison with exp. values: Note that there is a constant shift, which is most likely from differences in the pure compound vapor pressures used in the prediction and those found in this experiment.

\section{References}

[1] A. Fredenslund, J. Gmehling, P. Rasmussen, Vapour Liquid Equilibria Using UNIFAC, Elsevier: Amsterdam (1977)

[2] J.M. Stubbs, B. Chen, J.J. Potoff, J.I. Siepmann, Fluid Phase Equil. 183/184 (2001) 301-309

[3] J.R. Errington, A.Z. Panagiotopoulos, J.Phys.Chem.B 103 (1999) 6314 - 6322

[4] M.G. Martin, J.I. Siepmann, J.Phys.Chem.B 102 (1998) 2569-2577

[5] S.K. Nath, F.A. Escobedo, J.J. de Pablo, I. Patramai, Ind. Eng. Chem. Res. 37 (1998) 3195-3202

[6] A. Klamt, J. Phys. Chem. 99 (1995) 2224

[7] A. Klamt, V. Jonas, T. Buerger, J.C.W. Lohrenz, J. Phys. Chem. A 102 (1998) 5074

[8] A. Klamt, F. Eckert, Fluid Phase Equilibria 172 (2000) 43

[9] F. Eckert, A. Klamt, AICHE Journal 48 (2002) 369-385

[10] A. Klamt, G. Schüürmann, J. Chem. Soc. Perkin Trans.2 (1993) 799

[11] A. D. Becke, Phys. Rev. A, 38, (1988) 3098

[12] J. P. Perdew, Phys. Rev. B, 33, (1986) 8822

[13] R. Ahlrichs, M. Bär, M. Häser, H. Horn and C. Kölmel, Chem. Phys. Letters 162 (1989) 165

[14] A. Schäfer, A. Klamt, D. Sattel, J.C.W. Lohrenz, F. Eckert, PCCP 2 (2000) 2187

[15] A. Klamt, G. Krooshof, R. Taylor, AICHE Journal 48 (2002) 2332-2349

[16] COSMOtherm program, Release C1.2, COSMOlogic GmbH\&CoKG, Leverkusen, Germany (2002)

[17] see http://www.cosmologic.de/contest.html

[18] Kang, J. W., K. -P. Yoo, H. Y. Kim, H. Lee, D. R. Yang and C. S. Lee, Korea Thermo physical Properties Databank (KDB), Department of Chemical Engineering, Korea University, Seoul, Korea (2000).

[19] Lide, D. R., CRC Handbook of Chemistry and Physics, 80th Ed., CRC Press, Boca Raton (2000). 